\title{
Structural Brain Development and Aggression: A Longitudinal Study in Late Childhood
}

\author{
Hannah Roberts ${ }^{1,2}$. Elena Pozzi ${ }^{2}$ - Nandita Vijayakumar ${ }^{3} \cdot$ Sally Richmond ${ }^{4}$ Katherine Bray Kats $^{2,5}$ Camille Deane $^{3}$. \\ Sarah Whittle 2,5
}

Accepted: 25 January 2021 / Published online: 18 February 2021

(C) The Psychonomic Society, Inc. 2021

\begin{abstract}
This longitudinal study examined the neurodevelopmental correlates of aggression in children, focusing on structural brain properties. A community sample of 110 (60 females) children participated at age 8 years and again at age 10 years. Brain structure was assessed by using magnetic resonance imaging (MRI), and parents reported on child aggression using the Child Behavior Checklist. Analyses examined the relationship between aggression and development of volume of subcortical regions, cortical thickness, and subcortical-cortical structural coupling. Females with relatively high aggression exhibited reduced right hippocampal growth over time. Across males and females, aggression was associated with amygdala- and hippocampal-cortical developmental coupling, with findings for amygdala-cortical coupling potentially indicating reduced top-down prefrontal control of the amygdala in those with increasing aggression over time. Findings suggest that aggressive behaviors may be associated with alterations in normative brain development; however, results were not corrected for multiple comparisons and should be interpreted with caution.
\end{abstract}

Keywords Childhood aggression $\cdot$ Hippocampus $\cdot$ Structural covariance $\cdot$ Structural magnetic resonance imaging $\cdot$ Sex difference

Aggression that begins in childhood and persists throughout youth is associated with numerous aversive outcomes later in life, including strained interpersonal relationships and increased victimization, increased suicide, criminal activity, and spousal abuse (Huesmann, Eron, Lefkowitz, \& Walder, 1984; Schwartz, McFadyen-Ketchum, Dodge, Pettit, \& Bates, 1999; Zhang et al., 2012). Understanding the neurobiological correlates of aggression in children is valuable in identifying

Hannah Roberts

rohannah@med.umich.edu

1 Department of Psychiatry and Addiction Center, University of Michigan, 4250 Plymouth Road, Ann Arbor, MI 48109, USA

2 Melbourne Neuropsychiatry Centre, Department of Psychiatry, The University of Melbourne and Melbourne Health, Melbourne, Australia

3 School of Psychology, Deakin University, Geelong, Australia

4 Turner Institute for Brain and Mental Health, School of Psychological Sciences, Monash University, Melbourne, Australia

5 Melbourne School of Psychological Sciences, The University of Melbourne, Melbourne, Australia its mechanisms and thus may contribute to early detection and prevention efforts. Studying aggression in a community sample may provide novel insights into the ways neural circuits are associated with behavior without the confounding influence of psychiatric disorders or injury (Thijssen et al., 2015).

Neuroimaging studies have most consistently demonstrated that aggression is associated with structural abnormalities within the amygdala and hippocampus, and frontal regions, including the orbitofrontal cortex (OFC), dorsolateral prefrontal cortex (dlPFC), and anterior cingulate cortex (ACC). First, the amygdala plays a critical role in processing emotionally salient events, emotional learning and responding, and in autonomic and behavioral fear mediation (Cardinal, Parkinson, Hall, \& Everitt, 2002; Lombardo et al., 2001). Aggression therefore is believed to be associated with amygdala structure via dysfunction in processes involved in regulating defensive behaviors, and those that evoke negative affective reactions (Gregg \& Siegel, 2001; LeDoux \& Pine, 2016; Potegal, 2012; Siever, 2008). Several studies have shown associations between aggression and decreased amygdala volume in healthy children and adults, as well as in populations with increased rates of aggressive behavior, such as those with conduct disorder (CD), antisocial personality disorder (ASPD), and other 
psychopathology (Fairchild et al., 2011; Huebner et al., 2008; Pardini, Raine, Erickson, \& Loeber, 2014; Thijssen et al., 2015).

The hippocampus has been suggested to play an important role in aggression (Gregg \& Siegel, 2001; Guillot, Roubertoux, \& Crusio, 1994; Prior, Schwegler, Marashi, \& Sachser, 2004; Sluyter, van Oortmerssen, de Ruiter, \& Koolhaas, 1996). Hippocampal structure is thought to be associated with aggression given its critical roles in emotional regulation and fear learning (Raine et al., 2004). Prior work has found aggression to be associated with reduced hippocampal volumes in a range of adult populations, including adult males and adults with borderline personality disorder (Nunes et al., 2009; Pardini et al., 2014; Zetzsche et al., 2007). However, there have been mixed findings in children and adolescents, with both null findings and positive associations (Thijssen et al., 2015; Visser et al., 2013).

The structures of the frontal lobe serve an important role in the regulation of aggression as well (Ameis et al., 2014; Potegal, 2012). The OFC, along with the dIPFC and ACC, are thought to play key roles in emotional and behavioral regulation via projections from the amygdala and other areas in the anterior temporal lobe (Schoenbaum, Setlow, Saddoris, \& Gallagher, 2003). Aggressive behavior has thus been linked to aberrant frontal lobe structure and function, notably the failure to appropriately regulate negative affective states (Potegal, 2012; Siever, 2008). Of note, two meta-analyses including community and psychiatric samples of aggressive youth and adults identified the OFC and ACC as areas of consistently reduced volume and function (Raschle, Menks, Fehlbaum, Tshomba, \& Stadler, 2015; Yang \& Raine, 2009).

Furthermore, aggressive behavior has been associated with a dysfunction in the circuitry between limbic structures and the frontal lobe, where the amygdala in particular triggers affective responses, and the structures of the frontal lobe cognitively inhibit these urges in consideration of social context and moral reasoning (Davidson, Putnam, \& Larson, 2000; Potegal, 2012; Siever, 2008). As such, it is relevant to consider how the structure of these regions covary in relation to expressions of aggressive behavior. Indeed, structural differences have been identified in the subcorticalprefrontal circuit in studies with children and adolescents with externalizing disorders, in adolescents with psychopathic traits, and in healthy children who exhibit high levels of aggression (Ameis et al., 2014; Bos et al., 2018; Sarkar et al., 2012).

Relatively little research has examined the neurological correlates of aggression in children, and those that have often use a sample with a mean age above 10 years old, potentially failing to capture critical changes in the brain in earlier childhood (Ameis et al., 2014; Ducharme et al., 2011; Visser et al., 2013; Walhovd, Tamnes, Østby, Due-Tønnessen, \& Fjell, 2012). No other research to our knowledge has longitudinally examined the association between brain morphology and aggression using a community sample of children in this age range. This strategy allows for the investigation of potential deviation from neurotypical development of the amygdala, hippocampus, structures of the prefrontal cortex, and patterns of limbic-prefrontal coupling in aggressive children. Prior research has demonstrated that longitudinal investigation of brain development is critical in revealing the neurobiological mechanisms of behavioral and mental health problems in children and adolescents and may uncover important insights that are not possible with cross-sectional research (Bos et al., 2018; Dennis, Humphreys, King, Thompson, \& Gotlib, 2019; Whittle, Vijayakumar, Simmons, \& Allen, 2019). Indeed, one prior study found that increased aggression was related to decreases in hippocampal volume, and positive developmental coupling of the amygdala and prefrontal cortex, from late childhood into adolescence (Bos et al., 2018). Interestingly, results were specific to aggression, and did not replicate using other externalizing variables (i.e., rule-breaking). While externalizing behaviors more broadly, which include both aggressive and rule-breaking behaviors, have been linked to alterations in brain development in children (Ameis et al., 2014; Muetzel et al., 2018), prior research has demonstrated specific effects (Bos et al., 2018). Thus, it is important to distinguish the specific neurobiological associations of the distinct facets of externalizing behaviors.

The present study investigated whether baseline and change in aggression, measured by the aggressive behavior subscale of the Child Behavior Checklist for Ages 6-18 (CBCL), predicted growth of the amygdala, hippocampus, prefrontal cortex, as well as subcortical-prefrontal structural coupling, in a large community-based sample of children over a period of 2 years ( $8-10$ years of age). Given normative increases in amygdala and hippocampal volumes, and normative decreases in prefrontal thickness across childhood and adolescence, we hypothesized that relatively high and increasing levels of aggression in children would be associated with reductions in amygdala and hippocampal volume, and reductions in prefrontal thinning, from ages 8 to 10 years (Bos et al., 2018; Herting et al., 2018; Wierenga et al., 2014). We also hypothesized that increased positive maturational coupling between the amygdala and prefrontal structures would be associated with increased aggression based on prior research (Bos et al., 2018). We investigated sex as a moderator in all analyses given past research showing sex differences in both aggressive behavior and brain development (Perry \& Ostrov, 2018: Visser et al., 2013). Finally, we investigated rule-breaking and attention problems to assess specificity of findings. 


\section{Methods}

\section{Participants}

The present sample was derived from the Families and Childhood Transition Study conducted in Melbourne, Australia. Participants included a community sample of children recruited from metropolitan neighborhoods within the higher tertile of socioeconomic disadvantage, the procedures of which are described in detail elsewhere (Simmons et al., 2017). Socioeconomic disadvantage was estimated using the Socio-Economic Indexes for Areas Index of Relative Socio-Economic Disadvantage (IRSD) scale. Genetic ancestry was obtained from DNA as described in Simmons et al. (2017). Children were invited to participate in two waves of data collection, approximately 18 months apart. Of relevance to the current study, at both waves, data collection involved a structural Magnetic Resonance Imaging (MRI) brain scan and the completion of the CBCL by a parent (Achenbach \& Ruffle, 2000). At both waves, exclusion criteria included children who had a history of head trauma or loss of consciousness, a history of developmental or intellectual disorders, and those with MRI contraindications. Written consent from a parent/guardian and verbal assent from children were attained at both waves.

Of the 163 children who participated in the larger longitudinal study, 155 had aggressive behavior data (measured with the CBCL aggressive behavior subscale, see below), 153 completed an MRI scan at Time 1 (T1) and 129 of these completed an MRI scan at Time 2 (T2). After accounting for poor quality MRI scans $(\mathrm{n}=7)$ and missing aggression data ( $\mathrm{n}$ $=6$ ), the final sample for imaging analyses comprised 110 children (60 females) who had available data at both waves. See Table 1 for demographic data. Our sample for imaging analyses did not significantly differ from the total sample $(n=$ 163 ) in age, sex, neighborhood disadvantage, or aggression scores (all $p>0.05$ ).

\section{Materials}

\section{Aggressive Behaviors}

A parent (mothers in most cases) report of children's aggression was obtained by using the CBCL at each time point. The CBCL has been recognized to have good psychometric properties, including demonstrated validity and reliability (Nakamura, Ebesutani, Bernstein, \& Chorpita, 2008). The CBCL aggressive behavior subscale demonstrates excellent clinical agreement with the diagnosis of disruptive behavior disorders including CD and ODD (Bellina et al., 2012). The aggressive behavior subscale contains 18 items scored on a 3 point Likert scale consisting of $0=$ "Not true (as far as you know)"; 1 = "Somewhat or sometimes true"; and 2 = "Very true or often true." While the aggressive behavior subscale was of primary interest for this study, rule breaking and attention problems subscales were also used to test specificity of effects. The attention problem subscale was utilized as a measure of generally disruptive and impulsive behavior, constructs which both somewhat overlap with CBCL externalizing subscales (Achenbach \& Rescorla, 2001). CBCL subscale $\mathrm{T}$-scores were used in all analyses. Note that use of raw $\mathrm{CBCL}$ subscale scores yielded very similar results.

\section{Image acquisition}

At each time point, imaging data were collected using a 3 Tesla Siemens TIM Trio scanner (Siemens, Erlangen, Germany) at the Murdoch Children's Research Institute in Melbourne, Australia. At both baseline and follow-up, children underwent a mock scan in a replica MRI prior to the commencement of the real scan. During the mock scan, children received information about the real MRI scan and were monitored for indications of anxiety as well as the ability to remain still. For actual scanning, children were laid in a supine position in a 32-channel head coil. Structural T1-weighted images had the following parameters, repetition time $=$

Table 1 Demographic information for imaging sample $(\mathrm{N}=110)$

\begin{tabular}{|c|c|c|c|c|c|c|}
\hline & \multicolumn{3}{|l|}{ Wave 1} & \multicolumn{3}{|c|}{ Wave 2} \\
\hline & Mean & SD & Range & Mean & SD & Range \\
\hline Age (yr) & 8.45 & 0.33 & $8.00-9.06$ & 9.98 & 0.35 & $9.41-10.82$ \\
\hline CBCL aggression subscale & 50.00 & 10.00 & 40.39- 95.41 & 50.00 & 10.00 & $41.93-95.66$ \\
\hline \multirow[t]{2}{*}{$\mathrm{SES}^{\mathrm{a}}$} & 40.31 & 24.00 & $1-95$ & - & - & - \\
\hline & European $(\%)$ & Asian $(\%)$ & & & & \\
\hline Ancestry & 94 & 6 & & & & \\
\hline
\end{tabular}

Note. CBCL aggression subscale values represent t-scores.

${ }^{a}$ Socio-Economic Indexes for Areas Index of Relative Socio-Economic Disadvantage (IRSD) scale. Scale reflects percentile, with higher scores indicating lower disadvantage. 
2,530 msec; echo time1 $=1.74 \mathrm{msec}$, echo time $2=3.6 \mathrm{msec}$, echo time $3=5.46 \mathrm{msec}$, echo time $4=7.32 \mathrm{msec}$; flip angle $=$ $7^{\circ}$, field of view $=256 \times 256 \mathrm{~mm}$. A total of 176 contiguous slices $1.00-\mathrm{mm}$ thick slices (voxel dimensions $=1.0 \mathrm{~mm}^{3}$ ) were obtained.

\section{Procedure}

\section{Image processing}

We focused on cortical thickness given that it is the primary driver of volumetric changes across development (Tamnes et al., 2017). Cortical thickness was estimated at each wave using the output from the longitudinal stream of FreeSurfer, version 5.3 (http://surfer.nmr.mgh. harvard.edu/). This process involves the creation of an average template onto which an unbiased image is reconstructed and segmented for each subject. Additionally, the hippocampus and amygdala volumes were estimated by using FreeSurfer's automated segmentation. Visual inspection of all longitudinally processed images was completed by a trained researcher blind to participant characteristics to ensure the satisfactory segmentation of gray/white matter and improve output quality. Manual corrections were made where there was improper segmentation on four or more image slices (29\% of time point 1 images, $36 \%$ of time point 2 images). Note that the need for manual correction was not related to aggression scores either at baseline ( $p=$ $0.112)$ or follow-up $(p=0.202)$. Images were analyzed on an SGI/Linux workstation.

\section{Subcortical analyses}

Left and right amygdala and hippocampus were investigated as separate hypothesis-driven ROIs. Linear regression models were used to assess whether aggression at baseline, as well as change in aggression scores, predicted change (i.e., development) in hippocampus and amygdala volumes using R 3.5.2 software. Change in aggression scores and development in each ROI volume was quantified as symmetrized percent change (SPC). The annual rate of change was adjusted for average values across time. Sex effects were tested by including a main effect and an interaction with aggression variables. All models included socioeconomic status (SES, based on IRSD score), genetic ancestry, and intracranial volume (ICV) as covariates of noninterest. A bootstrapping procedure was performed (100 iterations) on models, which included interactive effects. Significant results were determined by using a $95 \%$ confidence interval based on bootstrap replicates.

\section{Cortical and amygdala-cortical coupling analyses}

Analyses were conducted in FreeSurfer's Query, Design, Estimate, and Contrast (QDEC) application to investigate whether baseline/change in aggression was associated with change in (i.e., development of) cortical thickness and whether sex moderated effects in follow-up analyses. To investigate whether baseline/change in aggression was associated with coupling of subcortical (amygdala and hippocampus) and cortical thickness development, interaction effects of baseline/ change in aggression by amygdala volume SPC (or hippocampal volume SPC) were examined. The moderating role of sex also was examined in models. Models examining interaction effects also included all lower-order terms of relevance. In addition, all models included SES and genetic ancestry as covariates of no interest. Change in cortical thickness was quantified as SPC. For all models, cluster-based correction for multiple comparisons was performed using a clusterdefining threshold of $p<0.001$, and a cluster-level threshold of $p<0.05$, implemented using Monte Carlo simulation $(10,000$ permutations $){ }^{32}$

Where significant effects were found in subcortical, corti$\mathrm{cal}$, and coupling analyses, simple slopes analyses were performed to understand the nature of the association between aggression and age-related changes in brain structure/coupling. Slopes were plotted at high and low levels (+1 SD and -1 SD from the mean/ CBCL subscale t-scores 40 and 60 , respectively) of the relevant variables in order to understand the nature of interaction effects. Note that while analyses were run with continuous CBCL values (due to the dimensional nature of behavioral problems like aggression), simple slopes analyses allowed us to make inferences about relatively higher versus lower levels of problems (Walton, Ormel, \& Krueger, 2011). For both subcortical ROI and whole brain thickness analyses, discriminant validity was evaluated by examining the main effects of both rule-breaking and attention problem CBCL subscale (baseline and change) scores, as well as their interactions with sex.

Before running analyses, all data were checked for statistical outliers, and a Winsorizing technique was used to replace 11 extreme brain volume values ( $>3 \mathrm{SD}$ of the sample mean).

\section{Results}

See Supplementary Figures S1 and S2 for an illustration of the range and changes in aggression scores from baseline to follow-up.

\section{Subcortical analyses}

There were no main effects of baseline aggression, aggression change, or sex moderated effects on left hippocampus 
development or left or right amygdala development (all $p$ values $>0.05$ ).

A significant two-way sex by baseline aggression interaction effect for right hippocampus development was found (Table 2). Follow-up analyses in each sex showed that the baseline aggression effect on right hippocampus development was significant in females $(B=-0.062, S E=0.020, p=0.004)$ but not males $(B=0.019, S E=0.026, p=0.475)$. For females, simple slopes analyses revealed significant age-related hippocampal development in those with low levels of aggression, but not in those with high levels of aggression. See Figure 1 for an illustration of the effect in females (and Table 3 for simple slopes statistics). See Supplementary Figure S3 for an illustration of the nonsignificant effect in males. Conversely, there were no effects of change in aggression (either main or sex moderated effects) on right hippocampus development (all $p$ values $>0.05$ ). A post-hoc test was performed to investigate whether hippocampal development was related to aggression at follow-up (and whether this association was moderated by sex); this analysis revealed nonsignificant effects (all $p$ values $>0.05$ ).

To examine the specificity of this finding to aggression, follow-up analyses were run to investigate whether baseline rule breaking or attention problems were associated with right hippocampal development. There were no significant main or sex moderated effects (all $p$ values $>0.05$ ).

\section{Cortical and amygdala-cortical coupling analyses}

There were no effects of baseline aggression or aggression change (either main effects or sex moderated effects) on change in cortical thickness in the left or right hemisphere. However, left amygdala development interacted with aggression change to predict development of thickness in the left lateral frontal cortex, temporal cortex, and precuneus (Table 4; Fig. 2). Simple slopes analyses showed that for all cortical regions, for children with relative reductions in aggression over time, low amygdala volume development (but not high amygdala volume development) was associated with reduced cortical thickness with age (see Fig. $2 b$ for an

Table 2 Regression of right hippocampal development on baseline aggression

\begin{tabular}{llll}
\hline & Effect & Standard Error & $p$ value \\
\hline Aggression & 0.026 & 0.024 & 0.281 \\
Sex & 3.94 & 0.170 & $0.022^{*}$ \\
SES & 0.006 & 0.007 & 0.431 \\
T1 ICV & $<0.001$ & $<0.001$ & 0.413 \\
Ancestry & 0.050 & 0.672 & 0.941 \\
Aggression x Sex & -0.077 & 0.033 & $0.023^{*}$ \\
\hline
\end{tabular}

Table 3 Simple slopes analysis of right hippocampal development and baseline aggression in females

\begin{tabular}{llll}
\hline Aggression & Slope & Standard error & $p$ value \\
\hline$-1 \mathrm{SD}$ & 52.645 & 11.014 & $<0.001$ \\
$+1 \mathrm{SD}$ & 6.924 & 11.428 & 0.567 \\
\hline
\end{tabular}

illustration of pars orbitalis effects). That is, children with relative reductions in aggression over time exhibited positive left amygdala-cortical developmental coupling (both amygdala and cortical regions exhibited greater reductions in size over time).

Regarding the hippocampus, left hippocampal development interacted with baseline aggression to predict development of thickness in left frontal, parietal, temporal, and posterior cingulate regions (Table 5; Fig. 3). Simple slopes analyses showed that for all cortical regions, for children with relatively high baseline aggression, low hippocampal volume development (but not high hippocampal volume development) was associated with reduced cortical thickness with age (see Fig. $3 \mathrm{~b}$ for an illustration of lateral orbitofrontal effects). That is, children with high baseline aggression exhibited positive left hippocampal-cortical developmental coupling.

To examine the specificity of these findings to aggression, follow-up analyses were run to investigate whether baseline/ change in rule breaking or attention problems were associated with developmental coupling of the left amygdala, left hippocampus, and cortical thickness. Change in both rule breaking and attention problems were both associated with developmental coupling of the left amygdala, and baseline rule breaking was associated with developmental coupling of the left hippocampus; however, the cortical regions implicated did not overlap with those implicated in aggression results (see Supplementary Material).

\section{Discussion}

In the present study, we investigated the neurodevelopmental correlates of aggressive behavior in a community-based sample of 110 children, aged 8 to 10 years. We found that aggressive behavior was associated with the development of the right hippocampus in females and the developmental coupling of the amygdala and hippocampus with frontal, temporal, and parietal cortices across males and females. Specifically, females with relatively low levels of aggressive behavior at baseline showed growth in the right hippocampus over time, whereas females with relatively high levels of aggressive behavior did not. Children with relatively low levels of aggressive behavior across time showed positive developmental coupling of amygdala and cortical structure over time. 


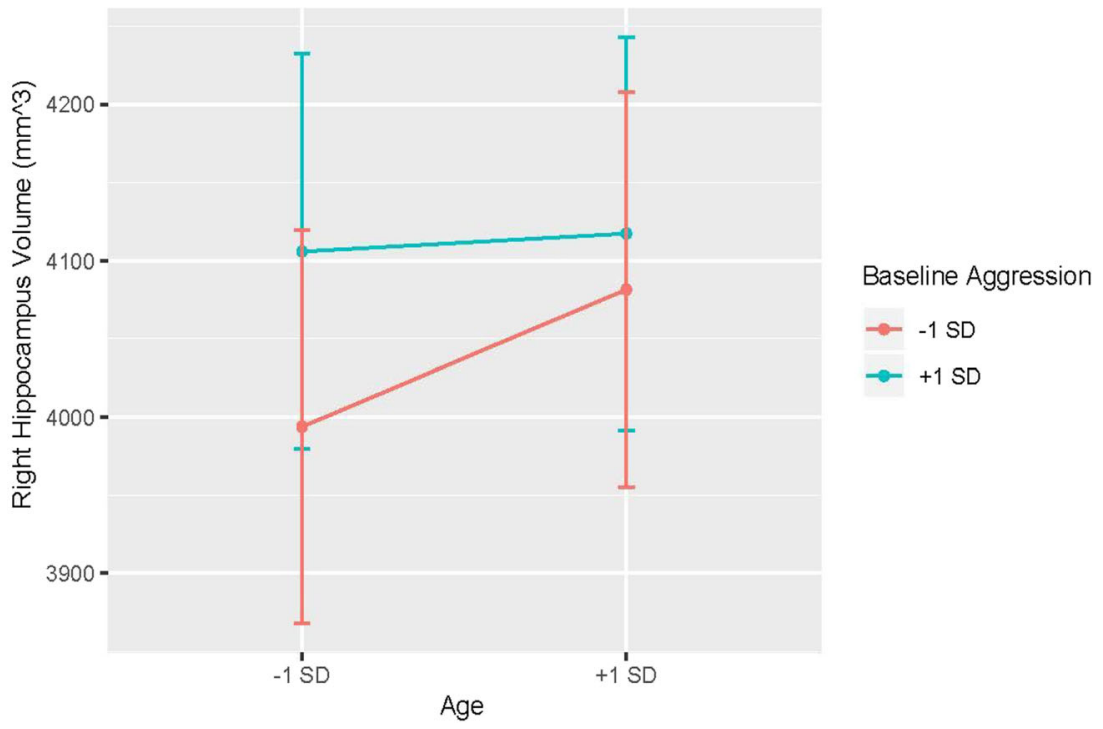

Fig. 1 Association between aggression and right hippocampal development in females. Simple slopes are plotted for + and -1 standard deviation from the mean for aggression score and age. *Significant slope $(p<0.05)$

Conversely, children with relatively high levels of aggressive behavior at baseline showed positive developmental coupling of the hippocampus and cortical structure over time. Development of the left hippocampus and bilateral amygdala were not associated with aggressive behaviors. Results were not corrected for multiple comparisons.

The only other study, to our knowledge, to examine longitudinally the relationship between hippocampal development and aggression in healthy children similarly identified a negative relationship, such that relatively decreased growth of the hippocampus was related to higher levels of aggression at baseline (Bos et al., 2018). Although no sex effects were found, this difference may in part be explained by the age of their sample, which ranged from 8 to 25 years old. Two prior cross-sectional studies have investigated the relationship between aggression and hippocampal volume in healthy children and have found either no relationship or increased hippocampal volume (Thijssen et al., 2015, Visser et al., 2013). However, these studies did not capture development and thus were unable to address potential age-related hippocampal effects.

Of note, we found that baseline aggression predicted hippocampal development, but hippocampal development did not predict levels of aggression at follow-up. This finding lends some support to an interpretation of aggression levels affecting brain development (and not vice versa), although it is possible that unmeasured variables contribute to both patterns of aggression and brain development.

In interpreting our sex effects for hippocampal development, it is important to note that physical aggression in girls at this age range is a relatively deviant social behavior (Fite, 2018). Interestingly, the hippocampus is a structure important in navigating normative social behavior (Rubin, Watson, Duff, \& Cohen, 2014). These processes are conducted largely via relational memory, flexible cognitions, and the ability to learn from consequences (Rubin et al., 2014). Although causality is not possible with longitudinal research, these changes in brain development may be interpreted through a Hebbian

Table 4 Left hemisphere regions whereby thickness development was predicted by left amygdala development and moderated by aggression change

\begin{tabular}{lllllll}
\hline Max & Size $\left(\mathrm{mm}^{\wedge} 2\right)$ & TalX & TalY & TalZ & CWP & Region \\
\hline-5.457 & 528.37 & -40.1 & 29.6 & 16.3 & 0.01740 & Rostral middle frontal \\
-4.824 & 522.96 & -7.0 & -40.0 & 25.9 & 0.01890 & Isthmus cingulate \\
-4.136 & 752.70 & -5.8 & -64.3 & 40.8 & 0.00140 & Precuneus \\
-4.052 & 1230.70 & -41.2 & -10.4 & -31.4 & 0.00010 & Inferior temporal \\
-3.788 & 726.08 & -34.4 & 48.4 & -9.9 & 0.00180 & Pars orbitalis \\
-3.517 & 1528.35 & -9.6 & 38.0 & 43.9 & 0.00010 & Superior frontal \\
-3.154 & 800.63 & -42.7 & -28.0 & 6.7 & 0.00090 & Transverse temporal \\
-2.826 & 679.18 & -51.9 & -3.1 & -13.3 & 0.00330 & Superior temporal \\
\hline
\end{tabular}




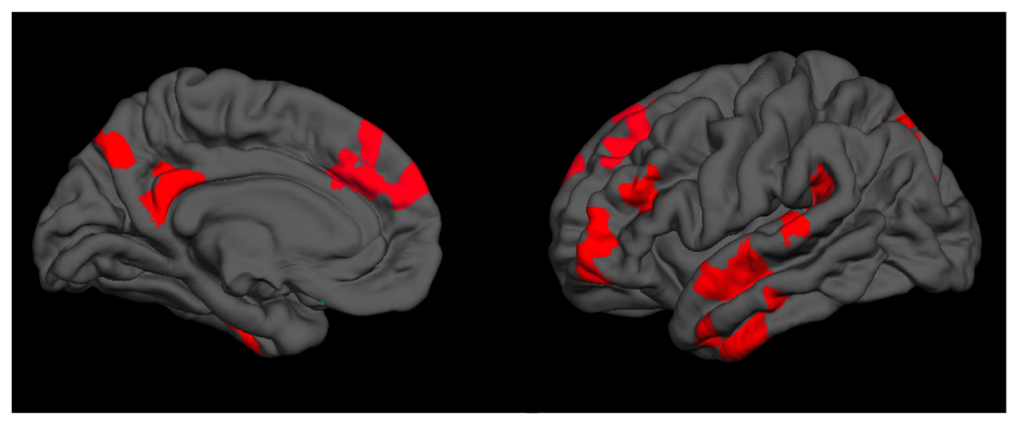

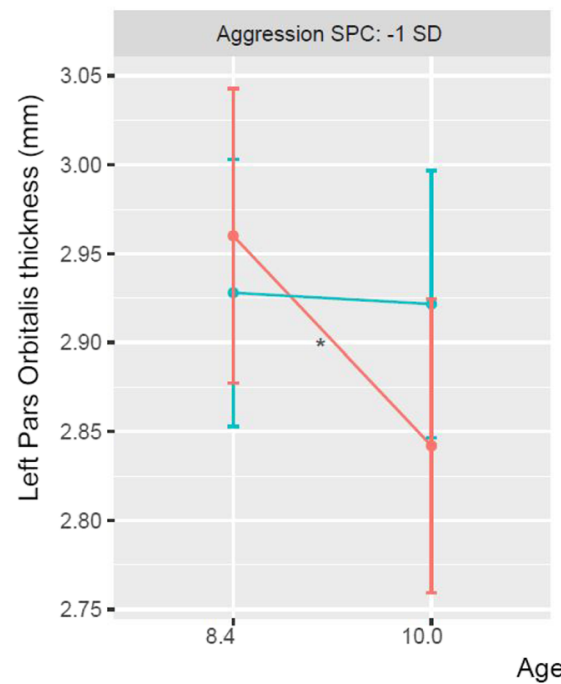

Fig. 2 a Left hemisphere regions whereby thickness development was predicted by left amygdala development and moderated by aggression change. b Developmental coupling of left pars orbitalis thickness and left amygdala volume in children with relative reductions (left) versus increases (right) in aggression over time (see Supplementary Figure S7 for plots of raw data). Only the age slope for children with relative

lens. Specifically, the more that girls engage in aggressive behavior, for whatever reason, the less they utilize the advanced social capabilities of the hippocampus. The less that girls utilize the hippocampus (i.e., relational memory, inhibition of aggressive responses based on learned information) in
Aggression SPC: +1 SD

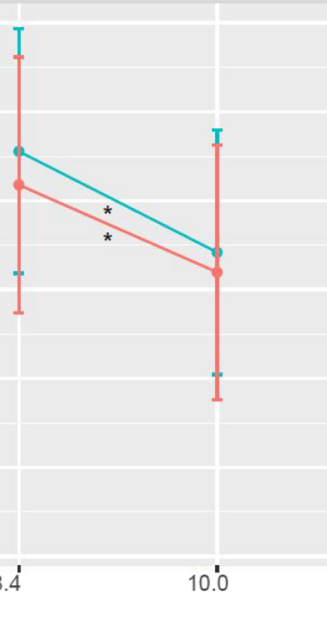

Left Amygdala SPC

$\rightarrow-1 S D$

$\rightarrow+1 S D$

aggression reductions and relatively more left amygdala volume development is not significant. Simple slopes are plotted for + and -1 standard deviation from the mean for amygdala development and age. Note that the pattern was identical for all cortical regions implicated. SPC $=$ symmetrized percent change

their social interactions, the weaker these neural associations become. Consequently, development of the underutilized hippocampus may be slowed compared to non-aggressive girls, who regularly call upon the hippocampus to navigate social relationships.

Table 5 Left hemisphere regions whereby thickness development was predicted by left hippocampal development and moderated by baseline aggression

\begin{tabular}{lllllll}
\hline Max & Size $\left(\mathrm{mm}^{\wedge} 2\right)$ & TalX & TalY & TalZ & CWP & Region \\
\hline 4.618 & 504.67 & -40.7 & -40.9 & -15.0 & 0.02570 & Fusiform \\
4.317 & 948.13 & -5.7 & -31.4 & 34.9 & 0.00010 & Posterior cingulate \\
4.264 & 1115.07 & -49.8 & -41.6 & 28.2 & 0.00010 & Supramarginal \\
4.230 & 997.14 & -34.5 & -76.9 & 24.4 & 0.00010 & Inferior parietal \\
3.593 & 487.76 & -33 & 34.9 & -8.4 & 0.03190 & Lateral orbitofrontal \\
3.358 & 652.76 & -44.8 & -59.6 & 3.0 & 0.00410 & Middle temporal \\
2.664 & 691.71 & -7.7 & 37.4 & 28.3 & 0.00310 & Superior frontal \\
2.530 & 567.58 & -43.8 & 28.4 & 26.5 & 0.01120 & Rostral middle frontal \\
\hline
\end{tabular}




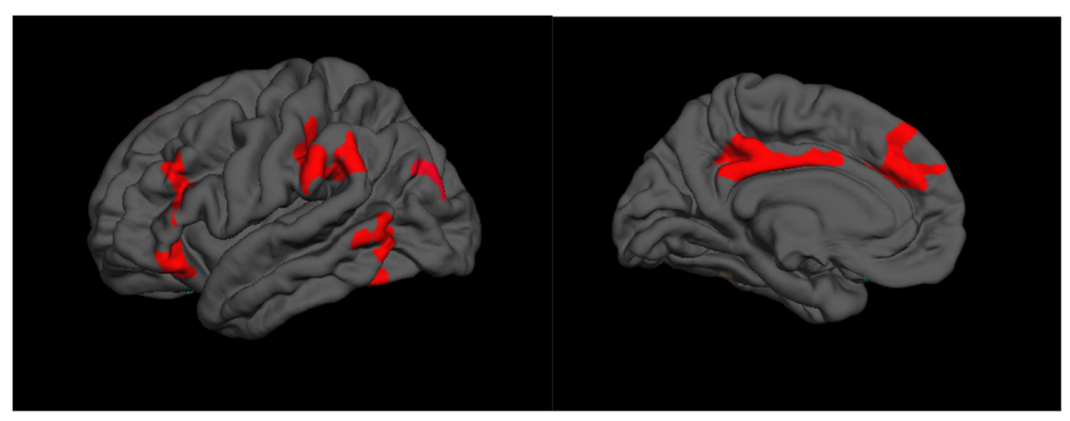

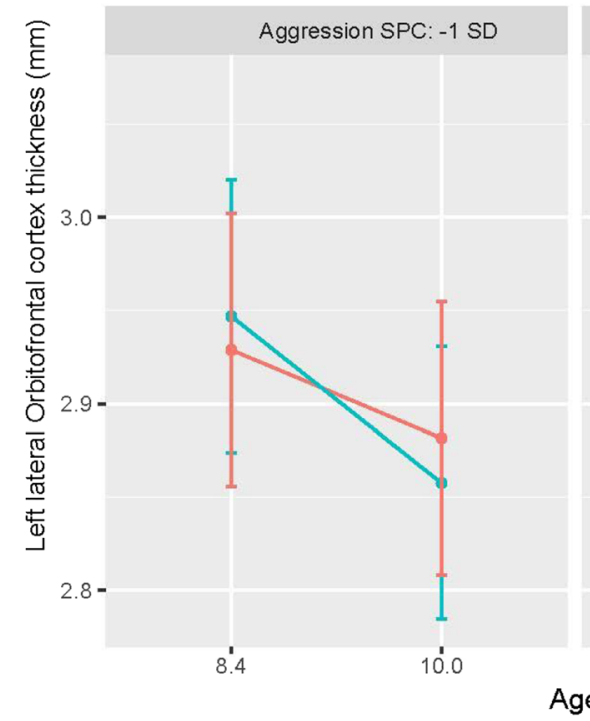

Fig. 3 a Left hemisphere regions whereby thickness development was predicted by left hippocampal development and moderated by baseline aggression. b Developmental coupling of left lateral orbitofrontal cortex thickness and left hippocampal volume in children with relative reductions (left) versus increases (right) in aggression over time (see Supplementary Figure S4 for plots of raw data). Only the age slope for

Regarding our failure to find associations between aggression and amygdala development, it is notable that other studies using community samples of children have found no association between bilateral amygdala volume and aggression or externalizing disorders (Ameis et al., 2014, Visser et al., 2013). Thijssen et al. (2015), conversely, was the first study to report an association between aggression and smaller amygdala volume in healthy children. Similar reductions in amygdala volume have been found in children with CD, psychopathic symptoms, and other externalizing psychopathology (Fairchild et al., 2011; Huebner et al., 2008; Pardini et al., 2014). Together, this leads us to speculate that abnormalities in amygdala volume as related to aggression are a result of comorbid affective or rule-breaking symptoms that would not be present in a community sample.

However, positive developmental coupling of the amygdala with frontal, temporal, and parietal cortex was identified in children with relatively low, but not high, levels of aggression. Only one longitudinal study to date has examined the relationship between subcortical-cortical maturational coupling and children with high baseline aggression and relatively more left hippocampal volume development is not significant. Simple slopes are plotted for + and -1 standard deviation from the mean for hippocampal development and age. Note that the pattern was identical for all cortical regions implicated. $\mathrm{SPC}=$ symmetrized percent change

youth externalizing behaviors (Bos et al., 2018). While it did not identify synchronous development of the amygdala and cortical structures in those with low levels of aggression, it is likely that the differences in findings can be largely attributed to coupling occurring beyond the age range found in the current study. Furthermore, the mean CBCL aggression score of the sample from Bos et al. (2018) was lower than in the current study, potentially truncating the ability to capture development of youth who show high levels of aggressive behaviors. Similarly, the only other study to our knowledge to investigate amygdala-cortical structural coupling specifically in relation to behavioral problems found that positive amygdala-OFC coupling was associated with lower externalizing problems in children and adolescents (Ameis et al., 2014). However, they did not find developmental (i.e., age) associations. Our finding of positive developmental coupling of the amygdala and prefrontal cortex in particular being associated with relatively lower aggression in males is somewhat consistent with previous work in an adolescent sample (Vijayakumar et al., 2017), where low levels of internalizing 
symptoms were found to be associated with positive developmental coupling between amygdala volume and prefrontal cortical thickness (Pozzi et al., 2019). Positive developmental coupling of these regions could indicate better affect regulation, potentially via top-down prefrontal control of the amygdala.

Positive developmental coupling of the amygdala and prefrontal cortex (i.e., greater reductions in cortical thickness combined with less increase in amygdala volume) has been found to be normative in previous work (Vijayakumar et al., 2017). Taken together, these results offer support for the existence of a normative pattern of positive coupling where the amygdala, which elicits aggressive responses, and cortical regions, including the prefrontal cortex, which cognitively moderates those urges, are synchronous in level of development. Therefore, developmental asynchrony may indicate poorer individual outcomes in terms of ability to make socially appropriate decisions.

In a pattern opposite to that of the amygdala, we found that children with relatively high, but not low, levels of aggression exhibited positive left hippocampal-cortical developmental coupling. The only longitudinal study to date exploring this relationship in the context of youth externalizing behaviors identified stronger hippocampal-ACC coupling in children with low, but not high, aggression scores (Bos et al., 2018). However, this study identified opposite patterns for other subcortical regions. In this, the authors call upon future research to examine whether maturational coupling in relation to externalizing behaviors is regionally specific. In interpreting our finding, it is important to appreciate the heterogeneity of the limbic system. While the amygdala has both afferent and efferent connections with the PFC, hippocampal connections are primarily efferent (Godsil, Kiss, Spedding, \& Jay, 2013). Unlike literature illustrating the anatomical and functional ties between the amygdala and the structures of the PFC, research in support of robust corticohippocampal associations is wanting. While research suggests the importance of hippocampal-PFC connectivity for cognition and emotion regulation, a comprehensive review of the role of the hippocampus in "conflict processing" points to the idea of a relatively autonomous hippocampus - one that has innate inhibitory abilities (Godsil et al., 2013; Ito \& Lee, 2016). As such, further research is needed to understand the structural coupling of the hippocampus with the PFC (and other cortical regions) in relation to aggression and other behaviors.

Some limitations should be noted. First, we did not correct for multiple comparisons, and as such, our findings may be subject to Type I error. However, given the absence of longitudinal imaging studies of aggression in children, our study represents an important contribution, and one that future studies should aim to replicate. Future longitudinal research would particularly benefit from extending the time frame of data collection, greater than the 18 months in the current study, in order to track changes that occur outside of the current age range. Another limitation regards the use of the CBCL aggressive behavior subscale, which was largely unable to capture relational aggression, as the majority of the items reflected either physical or verbal aggression, or were not able to be differentiated into a single subtype of aggression. Similarly, it could be argued that the CBCL aggressive behavior subscale encompasses constructs other than aggression, including disruptive and affective symptoms. While these items may seemingly contradict some definitions of aggression, the CBCL remains a widely used, validated, and reliable scale with excellent clinical utility and demonstrated ability in significantly predicting all disruptive behavior disorders (Bellina et al., 2012; Gomez, Vance, \& Gomez, 2014). Furthermore, our findings were specific to aggression, with null or different patterns of results found for rule-breaking and attention problems. In addition, we were unable to differentiate between proactive and reactive aggression using the $\mathrm{CBCL}$, which has meaningful biological correlates, including impacts on amygdala structure (Pardini et al., 2014). Of note, observational measures of aggression would be of considerable value in future research (Ostrov \& Hart, 2012). Finally, FreeSurfer version 5.3 was unable to estimate cerebellum volume using automated segmentation procedures. Future neuroimaging studies utilizing FreeSurfer should heed subsequent software updates.

To our knowledge, our study is the first structural imaging study to investigate aggression in nonclinical youth of this age range longitudinally. We tentatively suggest that aggression may predict attenuations in right hippocampal growth in females, whereas increases in aggression with age occur in parallel with attenuation of positive amygdala-prefrontal coupling in children. According to Moffitt's theory of life-course-persistent aggression, children that exhibit high levels of aggression early in life are likely to persist throughout the lifespan and predict future psychological, social, and legal consequences (Moffitt, 1993). Thus, it is of the utmost importance that aggressive behaviors are addressed early on to prevent attenuation in normative brain development as well as adverse consequences later in life.

Future studies should investigate aggression and brain development across both early childhood and adolescence in order to represent fully the rapidly changing social environments, as well as age-related brain development, across youth Future studies also should investigate brain developmentaggression associations using validated and reliable measures of physical, relational, and verbal aggression, as well as proactive and reactive aggression. Separating aggression into distinct constructs will illuminate the relationship between each distinct form and function of aggression without the confounding influence of disruptive or affective symptoms.

Supplementary Information The online version contains supplementary material available at https://doi.org/10.3758/s13415-021-00871-3. 


\section{References}

Achenbach, T., \& Ruffle, T. (2000). The Child Behavior Checklist and Related Forms for Assessing Behavioral/Emotional Problems and Competencies. Pediatrics in Review, 21(8),265-271. https://doi.org/ 10.1542/pir.21-8-265

Achenbach, T. M., \& Rescorla, L.A. (2001). Manual for the ASEBA School-Age Forms \& Profiles. Burlington: University of Vermont, Research Center for Children, Youth, \& Families.

Ameis, S., Ducharme, S., Albaugh, M., Hudziak, J., Botteron, K., \& Lepage, C. et al. (2014). Cortical Thickness, Cortico-Amygdalar Networks, and Externalizing Behaviors in Healthy Children. Biological Psychiatry, 75(1), 65-72. https://doi.org/10.1016/j. biopsych.2013.06.008

Bellina, M., Brambilla, P., Garzitto, M., Negri, G., Molteni, M., \& Nobile, M. (2012). The ability of CBCL DSM-oriented scales to predict DSM-IV diagnoses in a referred sample of children and adolescents. European Child \& Adolescent Psychiatry, 22(4), 235246. https://doi.org/10.1007/s00787-012-0343-0

Bos, M., Wierenga, L., Blankenstein, N., Schreuders, E., Tamnes, C., \& Crone, E. (2018). Longitudinal Structural Brain Development and Externalizing Behavior in Adolescence. Journal of Child Psychology And Psychiatry, 59(10), 1061-1072. https://doi.org/10. 1111/jcpp.12972

Cardinal, R., Parkinson, J., Hall, J., \& Everitt, B. (2002). Emotion and motivation: the role of the amygdala, ventral striatum, and prefrontal cortex. Neuroscience \& Biobehavioral Reviews, 26(3), 321-352. https://doi.org/10.1016/s0149-7634(02)00007-6

Davidson, R., Putnam, K., \& Larson, C. (2000). Dysfunction in the Neural Circuitry of Emotion Regulation-A Possible Prelude to Violence. Science, 289(5479), 591-594. https://doi.org/10.1126/ science.289.5479.591

Dennis, E., Humphreys, K., King, L., Thompson, P., \& Gotlib, I. (2019). Irritability and Brain Volume in Adolescents: Cross-Sectional and Longitudinal Associations. Social Cognitive And Affective Neuroscience. https://doi.org/10.1093/scan/nsz053.

Ducharme, S., Hudziak, J., Botteron, K., Ganjavi, H., Lepage, C., \& Collins, D. et al. (2011). Right Anterior Cingulate Cortical Thickness and Bilateral Striatal Volume Correlate with Child Behavior Checklist Aggressive Behavior Scores in Healthy Children. Biological Psychiatry, 70(3), 283-290. https://doi.org/10. 1016/j.biopsych.2011.03.015

Fairchild, G., Passamonti, L., Hurford, G., Hagan, C., von dem Hagen, E., \& van Goozen, S. et al. (2011). Brain Structure Abnormalities in Early-Onset and Adolescent-Onset Conduct Disorder. American Journal Of Psychiatry, 168(6), 624-633. https://doi.org/10.1176/ appi.ajp.2010.10081184

Fite, P. (2018). Developmental trajectories of relational aggression. In: S. Coyne and J. Ostrov, ed., The Development of Relational Aggression., 1st ed. New York: Oxford University Press, pp.49-60.

Godsil, B., Kiss, J., Spedding, M., \& Jay, T. (2013). The hippocampalprefrontal pathway: The weak link in psychiatric disorders? European Neuropsychopharmacology, 23(10), 1165-1181. https:// doi.org/10.1016/j.euroneuro.2012.10.018

Gomez, R., Vance, A., \& Gomez, R.M. (2014). Analysis of the Convergent and Discriminant Validity of the CBCL, TRF, and YSR in a Clinic-Referred Sample. Journal Of Abnormal Child Psychology, 42(8), 1413-1425. https://doi.org/10.1007/s10802014-9879-4

Gregg, T., \& Siegel, A. (2001). Brain structures and neurotransmitters regulating aggression in cats: implications for human aggression. Progress In Neuro-Psychopharmacology And Biological Psychiatry, 25(1), 91-140. https://doi.org/10.1016/s0278-5846(00) 00150-0
Guillot, P., Roubertoux, P., \& Crusio, W. (1994). Hippocampal mossy fiber distributions and intermale aggression in seven inbred mouse strains. Brain Research, 660(1), 167-169. https://doi.org/10.1016/ 0006-8993(94)90852-4

Herting, M., Johnson, C., Mills, K., Vijayakumar, N., Dennison, M., \& Liu, C. et al. (2018). Development of subcortical volumes across adolescence in males and females: a multisample study of longitudinal changes. Neuroimage, 172, 194-205. https://doi.org/10.1016/j. neuroimage.2018.01.020

Huebner, T., Vloet, T., Marx, I., Konrad, K., Fink, G., Herpertz, S., \& Herpertz-Dahlmann, B. (2008). Morphometric Brain Abnormalities in Boys With Conduct Disorder. Journal Of The American Academy Of Child \& Adolescent Psychiatry, 47(5), 540-547. https://doi.org/ 10.1097/chi.0b013e3181676545

Huesmann, L., Eron, L., Lefkowitz, M., \& Walder, L. (1984). Stability of aggression over time and generations. Developmental Psychology, 20(6), 1120-1134. https://doi.org/10.1037/0012-1649.20.6.1120

Ito, R., \& Lee, A. (2016). The role of the hippocampus in approachavoidance conflict decision-making: Evidence from rodent and human studies. Behavioural Brain Research, 313, 345-357. https://doi. org/10.1016/j.bbr.2016.07.039

LeDoux, J., \& Pine, D. (2016). Using Neuroscience to Help Understand Fear and Anxiety: A Two-System Framework. American Journal Of Psychiatry, 173(11), 1083-1093. https://doi.org/10.1176/appi.ajp. 2016.16030353

Lombardo, K., Herringa, R., Balachandran, J., Hsu, D., Bakshi, V., Roseboom, P., \& Kalin, N. (2001). Effects of acute and repeated restraint stress on corticotropin-releasing hormone binding protein mRNA in rat amygdala and dorsal hippocampus. Neuroscience Letters, 302(2-3), 81-84. https://doi.org/10.1016/s0304-3940(01) 01680-9

Moffitt, T. (1993). Adolescence-limited and life-course-persistent antisocial behavior: A developmental taxonomy. Psychological Review, 100(4), 674-701. https://doi.org/10.1037//0033-295x.100.4.674

Muetzel, R., Blanken, L., van der Ende, J., El Marroun, H., Shaw, P., \& Sudre, G. et al. (2018). Tracking Brain Development and Dimensional Psychiatric Symptoms in Children: A Longitudinal Population-Based Neuroimaging Study. American Journal Of Psychiatry, 175(1), 54-62. https://doi.org/10.1176/appi.ajp.2017. 16070813

Nakamura, B., Ebesutani, C., Bernstein, A., \& Chorpita, B. (2008). A Psychometric Analysis of the Child Behavior Checklist DSMOriented Scales. Journal Of Psychopathology And Behavioral Assessment, 31(3), 178-189. https://doi.org/10.1007/s10862-0089119-8

Nunes, P., Wenzel, A., Borges, K., Porto, C., Caminha, R., \& de Oliveira, I. (2009). Volumes of the Hippocampus and Amygdala in Patients With Borderline Personality Disorder: A Meta-Analysis. Journal Of Personality Disorders, 23(4), 333-345. https://doi.org/10.1521/pedi. 2009.23.4.333

Ostrov, J., \& Hart, E. (2012). Observational methods. In T. D. Little (Ed.), Oxford handbook of quantitative methods (Vol. 1, pp. 286-304). New York: Oxford University Press.

Pardini, D., Raine, A., Erickson, K., \& Loeber, R. (2014). Lower Amygdala Volume in Men is Associated with Childhood Aggression, Early Psychopathic Traits, and Future Violence. Biological Psychiatry, 75(1), 73-80. https://doi.org/10.1016/j. biopsych.2013.04.003

Perry, K., \& Ostrov, J. (2018). The Reciprocal Relations Between Female and Male Play Partners and Aggression in Early Childhood. Child Development, 90(1), 127-135. https://doi.org/10.1111/cdev.13178

Potegal, M. (2012). Temporal and frontal lobe initiation and regulation of the top-down escalation of anger and aggression. Behavioural Brain Research, 231(2), 386-395. https://doi.org/10.1016/j.bbr.2011.10. 049 
Pozzi, E., Bousman, C., Simmons, J., Vijayakumar, N., Schwartz, O., \& Seal, M. et al. (2019). Interaction between hypothalamic-pituitaryadrenal axis genetic variation and maternal behavior in the prediction of amygdala connectivity in children. Neuroimage, 197, 493501. https://doi.org/10.1016/j.neuroimage.2019.05.013

Prior, H., Schwegler, H., Marashi, V., \& Sachser, N. (2004). Exploration, emotionality, and hippocampal mossy fibers in nonaggressive $\mathrm{AB} /$ Gat and congenic highly aggressive mice. Hippocampus, 14(1), 135-140. https://doi.org/10.1002/hipo.10166

Raine, A., Ishikawa, S., Arce, E., Lencz, T., Knuth, K., \& Bihrle, S. et al. (2004). Hippocampal structural asymmetry in unsuccessful psychopaths. Biological Psychiatry, 55(2), 185-191. https://doi.org/10. 1016/s0006-3223(03)00727-3

Raschle, N., Menks, W., Fehlbaum, L., Tshomba, E., \& Stadler, C. (2015). Structural and Functional Alterations in Right Dorsomedial Prefrontal and Left Insular Cortex Co-Localize in Adolescents with Aggressive Behaviour: An ALE Meta-Analysis. PLOS ONE, 10(9), e0136553. https://doi.org/10.1371/journal.pone. 0136553

Rubin, R., Watson, P., Duff, M., \& Cohen, N. (2014). The role of the hippocampus in flexible cognition and social behavior. Frontiers In Human Neuroscience, 8. https://doi.org/10.3389/fnhum.2014. 00742

Sarkar, S., Craig, M., Catani, M., Dell'Acqua, F., Fahy, T., Deeley, Q., \& Murphy, D. (2012). Frontotemporal white-matter microstructural abnormalities in adolescents with conduct disorder: a diffusion tensor imaging study. Psychological Medicine, 43(02), 401-411. https://doi.org/10.1017/s003329171200116x

Schoenbaum, G., Setlow, B., Saddoris, M., \& Gallagher, M. (2003). Encoding Predicted Outcome and Acquired Value in Orbitofrontal Cortex during Cue Sampling Depends upon Input from Basolateral Amygdala. Neuron, 39(5), 855-867. https://doi.org/10.1016/s08966273(03)00474-4

Schwartz, D., McFadyen-Ketchum, S.A., Dodge, K.A., Pettit, G.S., \& Bates, J.E. (1999). Early behavior problems as a predictor of later peer group victimization: Moderators and mediators in the pathways of social risk. Journal of Abnormal Child Psychology, 27, 191-201.

Siever, L. (2008). Neurobiology of Aggression and Violence. American Journal Of Psychiatry, 165(4), 429-442. https://doi.org/10.1176/ appi.ajp.2008.07111774

Simmons, J., Schwartz, O., Bray, K., Deane, C., Pozzi, E., \& Richmond, S. et al. (2017). Study protocol: families and childhood transitions study (FACTS) - a longitudinal investigation of the role of the family environment in brain development and risk for mental health disorders in community based children. BMC Pediatrics, 17(1). https://doi.org/10.1186/s12887-017-0905-x

Sluyter, F., van Oortmerssen, G., de Ruiter, A., \& Koolhaas, J. (1996). Aggression in wild house mice: Current state of affairs. Behavior Genetics, 26(5), 489-496. https://doi.org/10.1007/bf02359753

Tamnes, C.K., Herting, M.M., Goddings, A.L., Meuwese, R., Blakemore, S.J., Dahl, R.E., Güroğlu, B., Raznahan, A., Sowell, E.R., Crone, E.A. and Mills, K.L., 2017. Development of the cerebral cortex across adolescence: a multisample study of inter-related longitudinal changes in cortical volume, surface area, and thickness. Journal of Neuroscience, 37(12), pp.3402-3412.

Thijssen, S., Ringoot, A., Wildeboer, A., Bakermans-Kranenburg, M., El Marroun, H., \& Hofman, A. et al. (2015). Brain morphology of childhood aggressive behavior: A multi-informant study in schoolage children. Cognitive, Affective, \& Behavioral Neuroscience, 15(3), 564-577. https://doi.org/10.3758/s13415-015-0344-9

Vijayakumar, N., Allen, N., Dennison, M., Byrne, M., Simmons, J., \& Whittle, S. (2017). Cortico-amygdalar maturational coupling is associated with depressive symptom trajectories during adolescence. Neuroimage, 156, 403-411.

Visser, T., Ohan, J., Whittle, S., Yücel, M., Simmons, J., \& Allen, N. (2013). Sex differences in structural brain asymmetry predict overt aggression in early adolescents. Social Cognitive And Affective Neuroscience, 9(4), 553-560. https://doi.org/10.1093/scan/nst013

Walhovd, K., Tamnes, C., Østby, Y., Due-Tønnessen, P., \& Fjell, A. (2012). Normal variation in behavioral adjustment relates to regional differences in cortical thickness in children. European Child \& Adolescent Psychiatry, 21(3), 133-140. https://doi.org/10.1007/ s00787-012-0241-5

Walton, K., Ormel, J., \& Krueger, R. (2011). The Dimensional Nature of Externalizing Behaviors in Adolescence: Evidence from a Direct Comparison of Categorical, Dimensional, and Hybrid Models. Journal Of Abnormal Child Psychology, 39(4), 553-561. https:// doi.org/10.1007/s10802-010-9478-y

Whittle, S., Vijayakumar, N., Simmons, J., \& Allen, N. (2019). Internalizing and Externalizing Symptoms Are Associated With Different Trajectories of Cortical Development During Late Childhood. Journal of The American Academy Of Child \& Adolescent Psychiatry. https://doi.org/10.1016/j.jaac.2019.04.006

Wierenga, L., Langen, M., Ambrosino, S., van Dijk, S., Oranje, B., \& Durston, S. (2014). Typical development of basal ganglia, hippocampus, amygdala and cerebellum from age 7 to 24 . Neuroimage, 96, 67-72. https://doi.org/10.1016/j.neuroimage.2014.03.072

Yang, Y., \& Raine, A. (2009). Prefrontal structural and functional brain imaging findings in antisocial, violent, and psychopathic individuals: A meta-analysis. Psychiatry Research: Neuroimaging, 174(2), 81-88. https://doi.org/10.1016/j.pscychresns.2009.03.012

Zetzsche, T., Preuss, U., Frodl, T., Schmitt, G., Seifert, D., \& Münchhausen, E. et al. (2007). Hippocampal volume reduction and history of aggressive behaviour in patients with borderline personality disorder. Psychiatry Research: Neuroimaging, 154(2), 157170. https://doi.org/10.1016/j.pscychresns.2006.05.010

Zhang, P., Roberts, R., Liu, Z., Meng, X., Tang, J., Sun, L., \& Yu, Y. (2012). Hostility, Physical Aggression and Trait Anger as Predictors for Suicidal Behavior in Chinese Adolescents: A School-Based Study. Plos ONE, 7(2), e31044. https://doi.org/10.1371/journal. pone.0031044

Publisher's note Springer Nature remains neutral with regard to jurisdictional claims in published maps and institutional affiliations. 